\title{
Breast cancer associated with paraneoplastic dermatomyositis (DM); case report
}

\author{
Abeer Hussien Anter ${ }^{1,2 *}$ and Samir Taha Alfotih ${ }^{1}$ \\ ${ }^{1}$ Oncology Department, King Fahad Hospital, Madinah, Saudi Arabia \\ ${ }^{2}$ Clinical Oncology and Nuclear Medicine Department, Faculty of Medicine, Mansoura University, Mansoura, Egypt
}

\section{Summary}

Breast cancer is the most common cancer in women. Dermatomyositis (DM) is an uncommon idiopathic inflammatory myopathy manifested by classic skin findings and proximal muscle weakness. However, DM may also manifest as a paraneoplastic syndrome of an underlying malignancy. Here, we report a case of a patient who presented with breast cancer and DM symptoms. The patient's rash and muscle weakness progressed during the first cycle of neoadjuvant chemotherapy, while she was already started on medical treatment of these symptoms with oral prednisone. Her cutaneous and musculoskeletal improved dramatically following the treatment of her breast cancer with surgery. Our case report describes the rapid progression and regression of her symptoms emphasizing the benefit of early diagnosis and treatment of DM as well as the underlying breast cancer.

\section{Background}

Dermatomyositis (DM) is an idiopathic inflammatory myopathy characterized by proximal muscle weakness, rash, and other systemic manifestations. The association between DM and malignancy is wellestablished, with $15 \%-30 \%$ of DM patients having an underlying malignancy [1-3]. Although the frequency of the various tumor types found in patients with DM but breast cancers appear to be the most common $[4,5]$.

We present this case as a reminder that a common disease (breast cancer) may present with uncommon features (DM as a paraneoplastic syndrome), and physicians must consider malignancy as the underlying systemic process when an adult patient presents with DM.

\section{Case presentation}

A 39 year Syrian woman, refereed to our institute with diagnosis with right breast cancer for further work up and management. She was 2 months post-delivery, when presented to us with painful right breast mass discovered during her pregnancy. Apart of that mass, patient complained of a violaceous skin rash on her face, started one month after the delivery followed by weakness in the proximal muscles. Her family history was positive for breast cancer in her mother. She was treated by dermatologist for her rash with oral prednisone and topical corticosteroids for a presumed atopic dermatitis, achieving a minimal improvement.

The clinical examination revealed, symmetrical erythematous macules over the upper eye led which became swollen. The rash extends to her cheeks (Figure 1). Another macular rash was noticed over the extensor surface of the hands and knees joints. Both arms were edematous with tenderness over the muscles without arthritis. Breast exam, reveled large mass occupying the whole Rt breast associated with peudoorange appearance, witha single large, mobile, pathologic lymph node was identified in the right axilla $(2 \times 3 \mathrm{~cm})$.

She underwent diagnostic mammogram and ultrasound that reported heterogeneous vascular mass at 12 o'clock to 6 o'clock measuring $9 \mathrm{~cm}$ in maximum diameter associated with skin thickening and abnormal axillary lymph nodes.

Then she underwent a core needle biopsy, which revealed a grade 3, triple-negative invasive ductal carcinoma. Metastatic work up was negative for distant metastasis.

Patient was reviewed by rheumatology team who diagnoses her as paraneoplastic dermatomyositis and started for her steroid. Given a diagnosis of locally advance Rt breast cancer with possible paraneoplastic dermatomyositis (DM), the recommendation was to start neoadjuvant chemotherapy adriamycin and cyclophosphamide (AC).

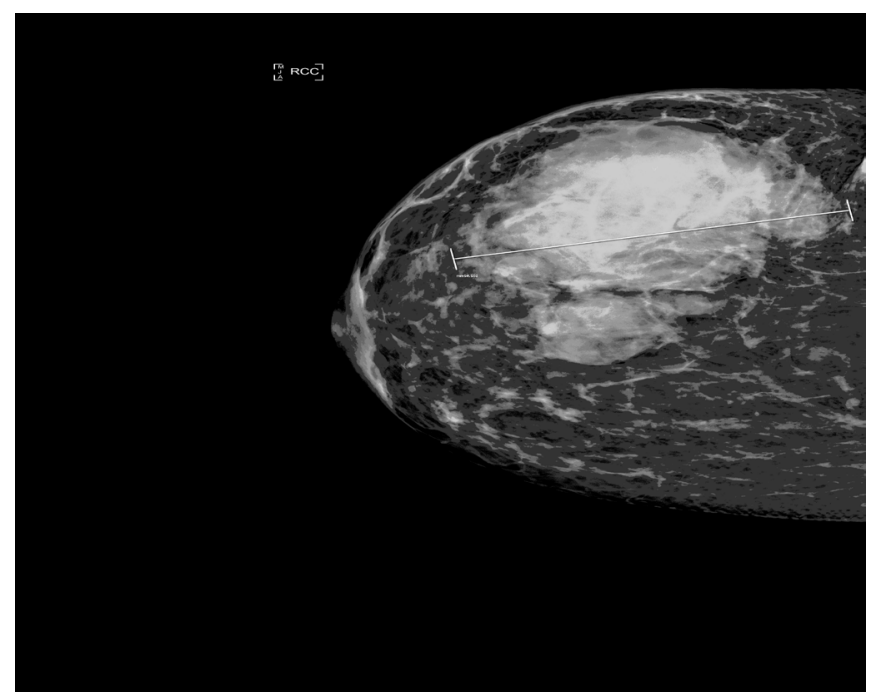

Figure 1. Right mammogram revealed a 9-cm irregular mass.

Correspondence to: Anter AH, Oncology Department, King Fahad Hospital, Madinah, Saudi Arabia, E-mail: abeerantar75@yahoo.com

Received: November 23, 2017; Accepted: December 22, 2017; Published: December 26, 2017 
One week after the first cycle of chemotherapy, she was presented to the emergency department, because of progressive proximal muscle weakness and dysphagia for solid food, the patient was admitted to hospital. Labs showed that AST and LDH increased 5 times and 2 times folds respectively whereas other liver enzymes were normal or mild elevation. Viral markers (HIV, HBV and HCV) were negative. All immune markers (ANA, Anti-DNA,Anti-SM, Anti-SSA, Anti-Scl-70) were negative. High dose of steroid was started by rheumatologist with minimal improvement. The breast tumor board and the rheumatologist recommendation were to proceed with surgery, hoping to control the DM.

She underwent mastectomy and axillary dissection without complication for a pT3N1M0 tumor. Out Of 18 axillary lymph nodes, 2 were positive for metastatic infiltrative duct carcinoma. After the surgery, the manifestations of DM started resolving, patient started to walk and to eat. She was treated with adjuvant Chemotherapy with doxorubicin and cyclophosphamide followed by docetaxel, she completed 7 cycle of her scheduled protocol but unfortunately before last one she presented to emergency department by Rt hypochondrial pain with elevated liver enzymes, admitted to hospital ,investigated by computed tomography for chest, abdomen and pelvis that showed multiple focal lesion in the liver and multiple bone metastasis in the vertebrae (Figure 2), she started supportive treatment but patient progressed rapidly and died from liver cell failure

\section{Discussion}

Breast cancer, mostly presents with palpable mass, abnormal mammogram during screening or symptoms related to metastases. It is very rare to present as paraneoplastic syndrome, like dermatomyositis (DM) as in our case.

Dermatomyositis (DM) is a rare autoimmune disease, with unknown etiology, in approximately $30 \%$ of DM associated with malignancy. Sigurgeirsson, et al. report incidence of $23 \%$ of malignancy in $392 \mathrm{DM}$ patients. Maoz, et al. find 37\% of $45 \mathrm{DM}$ patient have malignancies. In other large study (618 patients) Hill, et al. find $32 \%$ have malignancy. Patomenonget, et al. report $10 \%$ incidence of malignancy in $2518 \mathrm{DM}$ patients. Wide spectrum of malignancies

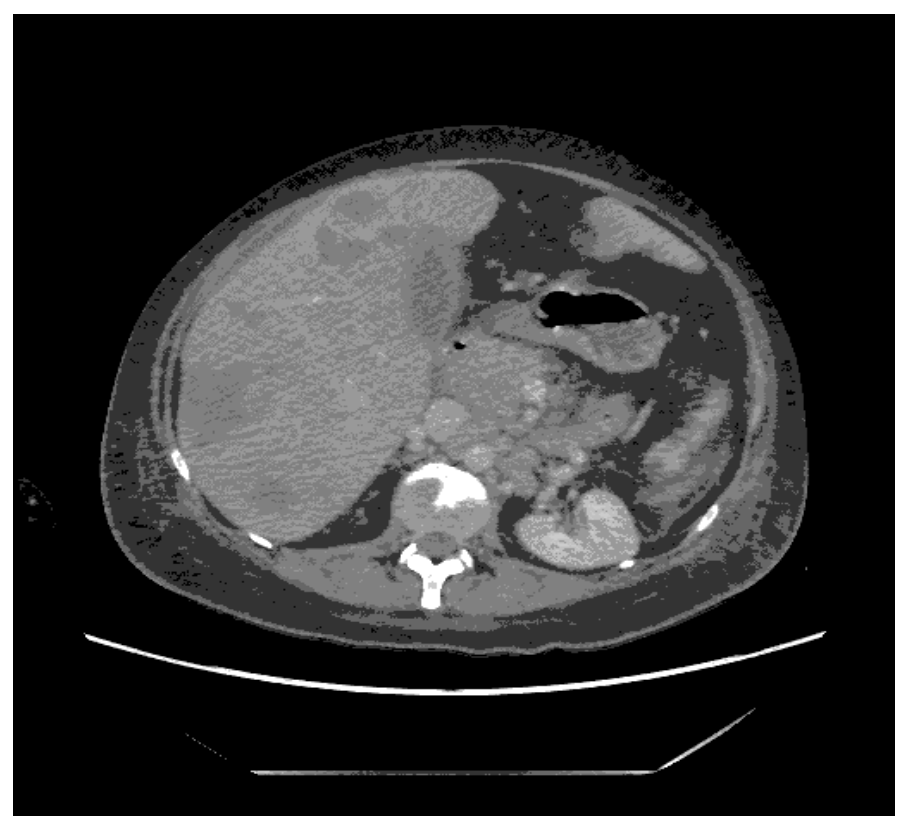

Figure 2. $\mathrm{CT}$ abdomenrevealed multiple focal lesions in liver and bone metastasis. diagnosed with DM, in approximately $20 \%$ the underlying malignancy is breast cancer [6]. In Asia the most common malignancy associated with DM are nasopharyngeal cancer and breast cancer in male and female respectively, whereas lung and ovarian cancer are the main malignancies in the Western patients [7-9].

Diagnosis of DM depends on 5 criteria defined by Bohan and Pete in 1975, which include clinical findings, EMG, raising muscle enzymes and muscle biopsy. The difference between the idiopathic DM and malignancy associated DM is potential to cure or at least to regress with treatment of the cancer. Diagnosis DM can proceed, concomitant or after diagnosis of malignancy. In our case, it was concomitantly diagnosed with breast cancer [10].

Up to our knowledge there is no lab test to differentiate between the idiopathic and the malignant associated DM.

No prospective data, how to manage those patients but in general we are following the standard guidelines for treatment of breast cancer, keeping in mind that involvement of the skin with the typical rash of $\mathrm{DM}$ is not equivalent to malignant skin involvement and that treatment of DM generally results in regression of the dermatologic abnormalities.

In patients with breast cancer and DM, the role of neoadjuvant chemo/hormonal therapy is debatable, and there are no data available in this setting. Wound healing after surgery is a concern if the skin condition is poor. When possible, we should improve the skin condition before surgical resection followed by adjuvant treatment for patients who are appropriate candidates for this approach. Alternatively, neoadjuvant chemo/hormonal therapy may be considered in appropriate cases if the skin is clear of infection [11-13].

In our case because of locally advanced breast cancer, we start neoadjuvant chemotherapy and because of flare of DM, we decided to go for surgery based on some reports that surgery may have advantage on neoadjuvant chemotherapy regarding rapid regression of the DM [11]. In our case despite the improvement in symptoms of DM, the patient had liver metastases causing liver failure and she died.

\section{References}

1. Olazagasti JM, Baez PJ, Wetter DA, Ernste FC (2015) Cancer risk in dermatomyositis: a meta-analysis of cohort studies. Am J Clin Dermatol 16: 89-98. [Crossref]

2. Richardson JB, Callen JP (1989) Dermatomyositis and malignancy. Med Clin North Am 73: 1211-1220. [Crossref]

3. Stockton D, Doherty VR, Brewster DH (2001) Risk of cancer in patients with dermatomyositis or polymyositis, and follow-up implications: a Scottish populationbased cohort study. Br J Cancer 85: 41-45. [Crossref]

4. Hill CL, Zhang Y, Sigurgeirsson B (2001) Frequency of specific cancer types in dermatomyositis and polymyositis:a population-based study. Lancet 357: 96-100.

5. Levine SM (2006) Cancer and myositis: new insights into an old association. Curr Opin Rheumatol 18: 620-624. [Crossref]

6. Callen JP, Hyla JF, Bole GG Jr, Kay DR (1980) The relationship of dermatomyositis and polymyositis to internal malignancy. Arch Dermatol 116: 295-298. [Crossref]

7. Yu KH, Wu YJ, Kuo CF, See LC, Shen YM, et al. (2011) Survival analysis of patients with dermatomyositis and polymyositis: analysis of 192 Chinese cases. Clin Rheumatol 30: 1595-1601. [Crossref]

8. Kuo CF, See LC, Yu KH (2011) "Incidence, cancer riskand mortality of dermatomyositis and polymyositis in Taiwan:a nationwide population study," BJD 165: 1273-1279.

9. Sigurgeirsson B, Lindelof B, Edhag O (1992) "Riskmof cancer in patients with dermatomyositis or polymyositis - apopulation-based study,". The New England Journal of Medicine 326: 363-367.

10. Voravud N, Dimopoulos M, Hortobagyi G, Ross M, Theriault R (1991) Breast cancer and second primary ovarian cancer in dermatomyositis. Gynecol Oncol 43: 286-290. [Crossref] 
11. Pectasides D1, Koumpou M, Gaglia A, Pectasides M, Lambadiari V, et al. (2006) Dermatomyositis associated with breast cancer. Anticancer Res 26: 2329-2331. [Crossref]

12. Barnes BE, Mawr B (1976) Dermatomyositis and malignancy. A review of the literature. Ann Intern Med 84: 68-76. [Crossref]
13. Ungprasert P, Leeaphorn N, Hosiriluck N (2013) Clinical Features of Inflammatory Myopathies and Their Association with Malignancy: A Systematic Review in Asian Population. ISRN Rheumatology.

Copyright: (C2017 Anter AH. This is an open-access article distributed under the terms of the Creative Commons Attribution License, which permits unrestricted use, distribution, and reproduction in any medium, provided the original author and source are credited. 